\title{
Failure Analysis to Unambiguously Clarify Hydrogen Fracturing in Steels
}

\author{
Pohl, Michael ${ }^{1}$ \\ 1. Ruhr-University Bochum, Faculty of Mechanical Engineering, Institute for Materials, Chair for \\ Material Testing, Bochum, Germany.
}

Hydrogen can get into metallic components and damage them in very different ways (fig. 1). In liquid phase metallurgical processes such as casting and welding, as a result of the dramatic reduction in the solubility of gases during solidification, gas bubbles can form causing porosity which particulary in conjunction with $\mathrm{H}$ induced cracking, form "fish-eyes" acting as dangerous internal stress raisers. This type of cracking, without the presence of porosity, which is also known as "flaking", can occur in thin steel wires only a few $\mu \mathrm{m}$ in diameter as well as in large 100 tonn steel forgings, in both cases significantly detrimentally affecting both, the manufacturing and service behavior of the component [1].

Apart from metallurgical sources, semi-finished and finished components can also absorb $\mathrm{H}$ from galvanic processes or as a result of corrosion. In these cases the $\mathrm{H}$ diffuses into regions of higher stress and strain causing cracking, often unexpectedly and after significant periods of time [2].

In failure analysis the clarification of hydrogen induced component damages is based on fractographic examinations in combination with $\mathrm{H}$-analysis.

The delayed fracture, which is typical for H-induced damages, is a strong evidence for a damage caused by hydrogen when analyzing damage. If a sample taken from the component breaks during a clamping test, this can only be caused by $\mathrm{H}$ which has always been present in the component. If too much of the hydrogen has escaped meanwhile, the fractographic comparison between a sample broken in a clamping test (fig.2) and the primary fracture of the component, as well as the final failure fracture (fig. $3 \mathrm{a}+\mathrm{b}$ ) is necessary. As these three fractures arise from the identical material, the comparison should lead to an unambiguous result.

Finally there is still the possibility to selectively detect the diffusible (damaging) hydrogen using the HCA-method [3].

The consecutive series of the investigation steps is shown schematically in figure 4.

\section{References:}

[1] M. Pohl, Hydrogen in Metals: A Systematic Overview, Pract. Metallogr. 51 (2014) p.291-305.

[2] M. Pohl, S. Kühn, Stress Corrosion Cracking of High Strength Steels, Materials Testing 52 (2010) p. $52-55$.

[3] S. Kühn, F. Unterumsberger, T. Suter, M. Pohl, New Methods to Analyse the Diffusible Hydrogen in High Strength Steels, Materials Testing 55 (2013) p. 648-652. 


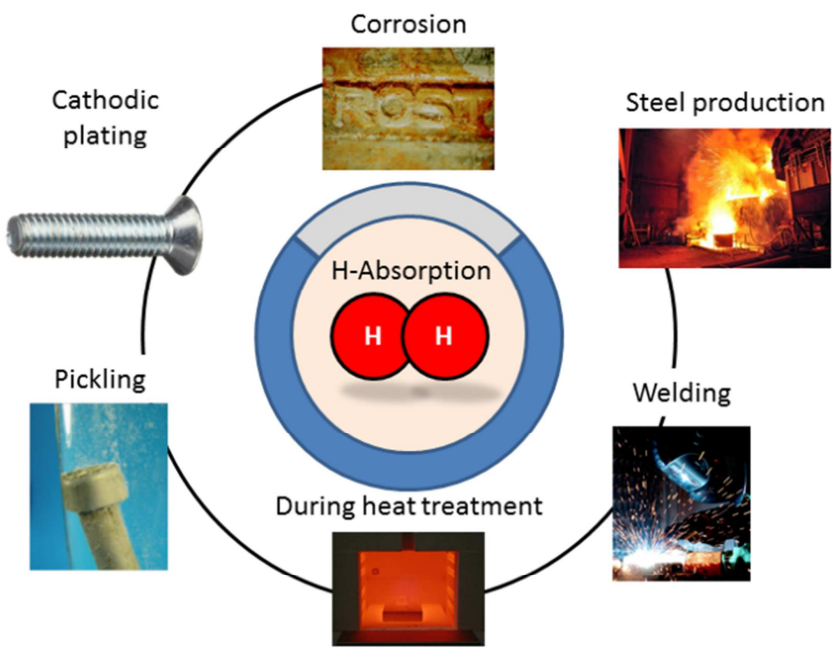

Operational hydrogen

Hydrogen due to manufacturing

Figure 1. Possible forms of H-Absorption.

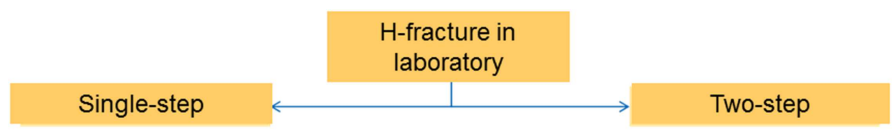

The sample is put into the tenter frame and into an acid bath afterwards.

The sample has to be taken out of the solution immediately after occurance of the delayed fracture!

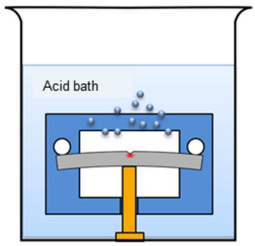

The sample is loaded in the acid bath for 5 to 15 minutes.

Afterwards a clamping test has to be made.

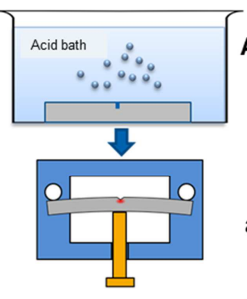

Advantage: microstructure of the fracture is not pit due to being taken out of the acid bath too late.

Figure 2. Generating a fracture for comparison.

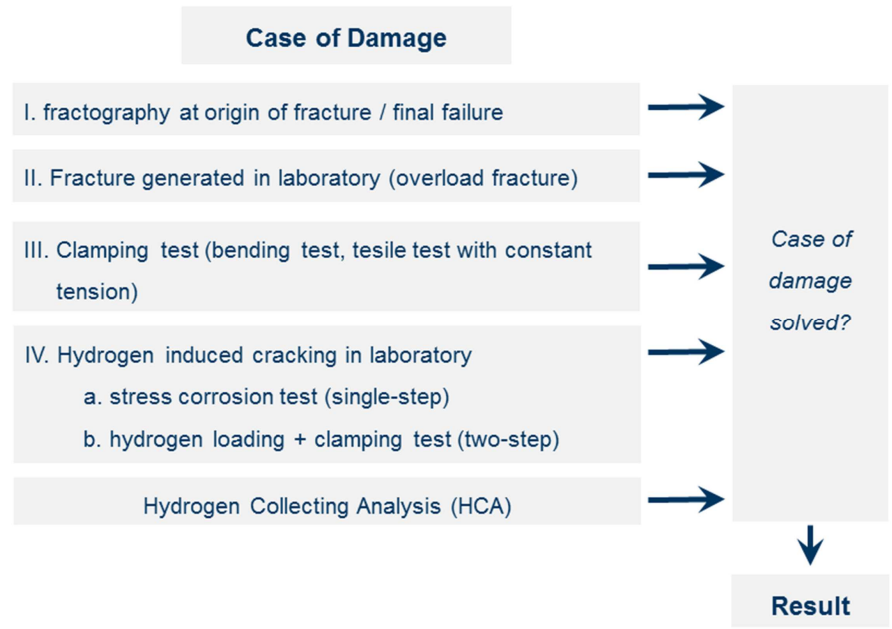

Figure 4. Unambiguously clarify $\mathrm{H}$-induced cracking.

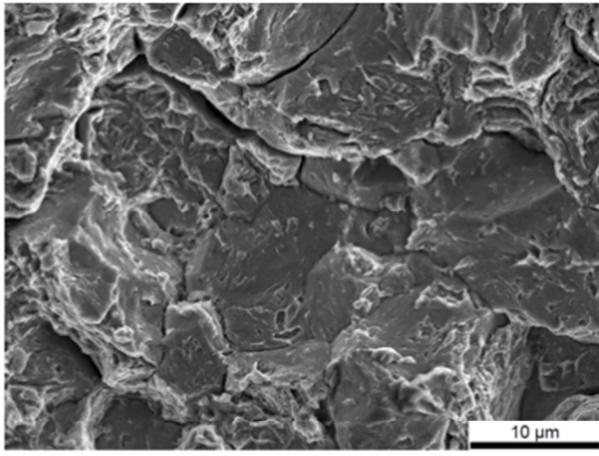

3a - Screw (12.09) broken while operating

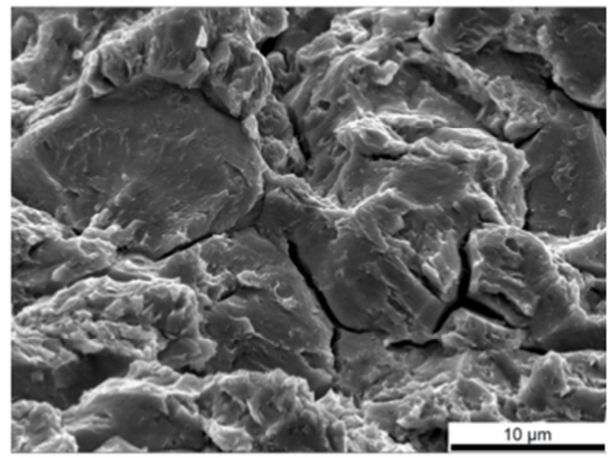

$3 \mathrm{~b}-\mathrm{H}$-induced fracture for comparison generated in laboratory

Figure 3. Generating a $\mathrm{H}_{2}-$ fracture for comparison. 